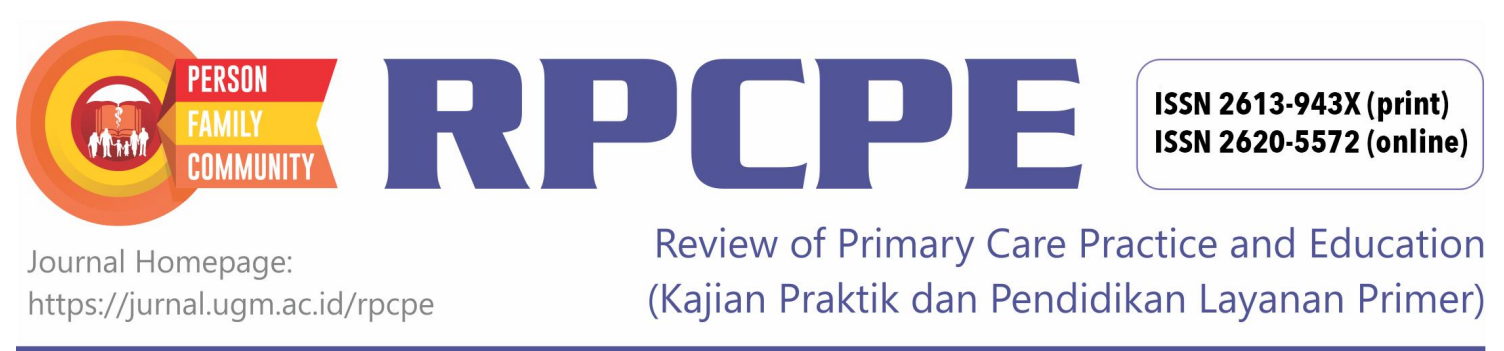

\title{
The Role of Primary Health Care in the Prevention of Noncommunicable Diseases
}

Indah S Widyahening ${ }^{1}$

\author{
${ }^{1}$ Department of Community Medicine; Faculty of Medicine; Universitas Indonesia; Indonesia \\ Corresponding Author: \\ Indah S Widyahening: Department of Community Medicine; Faculty of Medicine; Universitas Indonesia, Jl. Salemba Raya No.6, \\ RW.5, Kenari, Kec. Senen, Kota Jakarta Pusat, Daerah Khusus Ibukota Jakarta - 10430, Indonesia \\ E-mail: indah_widyahening@ui.ac.id
}

To cite this article:

Widyahening IS. The role of primary health care in the prevention of noncommunicable diseases. Rev Prim Care Prac and Educ. 2019; 2(1): 5-7.

\section{INTRODUCTION}

Noncommunicable diseases (NCDs) currently cause more deaths than all other causes combined. Cardiovascular diseases, cancer, chronic respiratory diseases and diabetes are the four major NCDs that are responsible for $82 \%$ of NCD deaths. Approximately $42 \%$ of all NCD deaths globally occurred before the age of 70 years, $48 \%$ of NCD deaths in the low- and middle-income countries and $28 \%$ in high-income countries were in individuals aged under 70 years $^{1}$.

Due to the chronic nature and the complications that arise, NCDs bring devastating social, economic and public health impact. The management of their various long-term complications accompanied by the typical existence of multimorbidity will give rise to an increased complexity of care in the future. Aging, improved medical therapies, demographic transition, life-style changes, globalization and urbanization are factors that are contributing to the increased prevalence, severity and complexity of NCDs.

As an anticipation to the expected increase in the burden of NCDs in the future, the World Health Organization (WHO) declared the global action plan for the prevention and control of noncommunicable diseases 2013-2020. In the planned strategy is the goal: "to strengthen and orient health systems to address the prevention and control of noncommunicable diseases and the underlying social determinants through people-centered primary health care and universal health coverage"2.

\section{Prevention of noncommunicable diseases}

Reducing the major modifiable behavioral risk factors, i.e. tobacco use, physical inactivity, unhealthy diet and the harmful use of alcohol, is the main focus in the prevention of NCDs. Among the global targets set by the WHO are a $30 \%$ relative reduction in prevalence of current tobacco use in persons aged $15+$ years, a $10 \%$ relative reduction in prevalence of insufficient physical activity, a $30 \%$ relative reduction in mean population intake of salt/sodium and at least $10 \%$ relative reduction in the harmful use of alcohol, as appropriate, within the national context ${ }^{2}$.

The most cost-effective strategies in achieving the goals should be a combination between policy, population and individual interventions ${ }^{3}$. Effective individual health-care interventions in the primary care level are considered to have a great potential to help reduce the burden of NCDs. Primary care as the health service entry point for the vast majority of most populations plays a key role in the delivery of prevention and care interventions for NCDs.

Since the prevention and management of NCDs require lifestyle and daily behavior change, emphasis must be upon the patient's central role and responsibility in health care. Therefore, the health care must be re-oriented around the patient and family. To successfully manage chronic conditions of NCDs, patients and families would further need services and support from their communities. Primary health care (PHC) is in the best position to provide those needs.

The characteristics of primary health care that are valuable in the prevention of NCDs

Starfield summarized the following ideal characteristics of primary care: comprehensive, coordinated, continuous, integrated, and accessible care ${ }^{4}$. Regarding the above characteristics, PHC is considered to be most suitable to address the challenges of chronic disease prevention and management ${ }^{5}$. 
"Comprehensive care" is the care that deals with a broad spectrum of health problems which encompass all stages of a patient's life cycle $e^{6,7}$. Although NCDs mainly occur during adulthood, it is now understood that for certain diseases, the process has begun as early as childhood ${ }^{8,9}$. Therefore, behavioral modification strategies as part of NCDs prevention should not only be targeted to those with ascertained risk factors but to all family members in the community using the life-course approach. Comprehensive care ensures that the major common risk factors of noncommunicable diseases (physical inactivity, obesity, poor nutrition, and tobacco use) have been addressed, and any health disparities in populations and their predisposing factors are considered, such as socioeconomic status. The care, which encompass all levels of prevention from health promotion to rehabilitation is provided for the general population as well as targets high-risk and priority populations in all the places in which members of the communities are found (schools, work sites, recreation areas, and religious and health care settings $)^{10}$.

"Coordinated care" ensures the supply of a mixture of health services and knowledge to fulfil a patient's needs. It conjointly refers to the affiliation between, or the rational ordering of, those services, including the resources of the community. Various medical management models consisting of complex elements specifically designed for chronic diseases have been developed ${ }^{11}$. One of the models that is included in the recommendations of American Diabetes Association as one of the strategies in improving care for people with diabetes is the Chronic Care Model ${ }^{12}$. The Chronic Care Model (CCM) works on the premise that good outcomes (better health status and patient satisfaction) result from productive interactions between patients, the healthcare practice, and the larger health system and other resources that exist in the community. The practice itself must be redesigned to be able to provide self-management support for the NCDs patients, appropriate care delivery system with task distribution among the multidisciplinary health care personnel, timely evidence-based decision support, which are guided by the optimal use of available clinical information systems ${ }^{13,14}$. The CCM emphasizes the central importance of PHCs and the recognition that the best clinical outcomes can be obtained when all model components are interconnected and working in a coordinated manner.

"Continuous care" refers to the well-rounded delivery of care by one individual or team of healthcare professionals ("clinician continuity") complemented by effective and timely maintenance and communication of health information (events, risks, advice, and patient preferences) ("record continuity"). Noncommunicable diseases are chronic conditions that require the provision of continuous care over an extended period (often lifelong). Collectively, health care personnel must to be able to provide the full spectrum of health care services, from clinical prevention through rehabilitation and end-of-life care.

"Integrated care" is intended to encompass the provision of comprehensive, coordinated, and continuous services that offer a seamless process of care. Integrated care is needed to care for chronic conditions in NCDs to ensure shared information across settings and providers, and across time (from the initial patient contact, onward). Integration also includes coordinating financing across different arms of health care (e.g., inpatient, outpatient, and pharmacy services), including prevention efforts, and incorporating community resources that can leverage overall health care services. The outcome of integrated services is improved health and wellbeing, less waste, less inefficiency and a less frustrating experience for patients ${ }^{15}$. Integrated management of NCDs is necessary because of the following reasons: most patients have more than one risk factor or type of NCDs (e.g. hypertension and obesity, diabetes and obesity, etc.), most NCDs place similar demands on health workers and health systems, and comparable ways of organizing care and managing these conditions are similarly effective regardless of etiology, and finally, most NCDs have common primary and secondary risk factors which could be addressed in one integrated effort ${ }^{14}$.

"Accessible care" refers to the convenience with which a patient can initiate an interaction for any health problem with a health care professional (e.g., by phone or at a treatment location) and includes efforts to eliminate barriers like those caused by geography, administrative hurdles, financing, culture, and language. Due to the longterm (often life-time) course of the NCDs, patients are in many ways their own primary care providers, therefore their needs and preferences must be considered in the development of management plans. The advantages of PHC centered approach include its relative proximity to the patients' homes which reduces travel costs; familiarity to the individual patients, families, and local communities by the health care personnel; the possibility of caring for defined populations which makes coverage and follow-up easier to assess and monitor; and better continuity of care, including that for comorbid illnesses ${ }^{5}$.

\section{CONCLUSIONS}

The core principals of the primary health care are considered to be well suited to address the burden of noncommunicable diseases. A comprehensive implementation of the Chronic Care Model where the primary health care has a central role to play as a coordination hub is recommended.

\section{REFERENCES}

1. World Health Organization. Global status report of noncommunicable diseases 2014. Geneva, Switzerland: World Health Organization. 2014.

2. World Health Organization. Global action plan for the prevention and control of noncommunicable diseases 2013-2020. Geneva: World Health Organization. 2013.

3. World Health Organization. Global status report on noncommunicable diseases 2010. Geneva: World Health Organization. 2011.

4. Starfield B. Is primary care essential?. Lancet 1994;344(8930):1129-33.

5. Beaglehole R, Epping-Jordan J, Patel V, Chopra M, Ebrahim S, Kidd $\mathrm{M}$, et al. Improving the prevention and management of chronic disease in low-income and middle-income countries: A priority for primary health care. Lancet. 2008;372:940-9.

6. Shi L. The impact of primary care: A focused review. Scientifica. 2012;2012(432892):1-22.

7. Oandasan IF, Archibald D, Authier L, Lawrence K, McEwen LA, 
Mackay MP, et al. Future practice of comprehensive care; Practice intentions of exiting family medicine residents in Canada. Canadian Family Physician. 2018;64(7):520-8.

8. Walker SP, Wachs TD, Gardner JM, Lozoff B, Wasserman GA, Pollitt E, et al. Child development: Risk factors for adverse outcomesin developing countries. Lancet 2007;369:145-57.

9. World Health Organization. Diet, nutrition and the prevention of chronic diseases: Report of a joint WHO/FAO expert consultation. Geneva: World Health Organization. 2002.

10. National Association of Chronis Disease Directors. Comprehensive and integrated chronic disease prevention: Action planning handbook for states and communities. Atlanta, Georgia: National Association of Chronis Disease Directors. 2005.

11. Grover A, Joshi A. An overview of chronic disease models: A systematic literature review. Global Journal of Health Science. 2015;7(2):210-27.

12. American Diabetes Association. Strategies for improving care. Diabetes Care. 2015;38(Suppl. 1):S5-S7.

13. Bodenheimer $\mathrm{T}$, Wagner $\mathrm{E}$, Grumbach $\mathrm{K}$. Improving primary care for patients with chronic illness: the chronic care model, part 2. JAMA. 2002;288:1909-14

14. Barceló A, Epping-Jordan J, Orduñez P, Luciani S, Agurto I, Tasca R. Innovative care for chronic conditions: Organizing and delivering high quality care for chronic noncommunicable diseases in the Americas. Washington DC: Pan American Health Organization. 2013.

15. World Health Organization. Innovative care for chronic conditions: building blocks for action: global report. Geneva: World Health Organization. 2002. 